Revista Ingeniería y Región. 2016;15(1):49-56

\title{
Viabilidad técnica para la creación de un complejo eólico generador de energía eléctrica en el departamento del Huila
}

\author{
Technical feasibility for the creation of a complex wind \\ power generator electrical in the department of Huila
}

\author{
Jesús David Quintero Polanco ${ }^{1}$, Johan Julián Molina Mosquera² y John Serrato
}

\begin{abstract}
Resumen
La humanidad se ve avocada actualmente en una crisis energética y ambiental, debido al sobrecalentamiento global generados por la producción de energías convencionales, por lo que se presenta una solución a mediano plazo a este problema, que en este caso será la creación de un parque eólico que ofrece un cambio en la energía racional, accesible y limpia; debido a que es una de las grandes preocupaciones de los países que firmaron el tratado de Kioto. Es por esto que Colombia tiene un alto potencial en recursos renovables, en la que se debe tomar conciencia del aprovechamiento adecuado y eficiente de todos estos recursos. El potencial energético de Colombia, es debido a la diversidad geográfica, la cual posee una variedad en sus recursos energéticos, si se logra aprovechar de manera eficiente todos los recursos, disminuiría en un porcentaje el sobrecalentamiento de la tierra.
\end{abstract}

La metodología empleada se ha basado en encontrar la mayor intensidad de viento que se presenta en la región del departamento del Huila basado en la investigación de datos que ha recopilado en registro el IDEAM por más de diez años.

Palabras clave: Meteorología; distribución; histograma; parámetros; rosa del viento; impacto ambiental

\begin{abstract}
Humanity is now doomed in an energy and environmental crisis, due to global overheating generated by the production of conventional energy, so a medium-term solution to this problem, which in this case will be the creation of a wind farm presents It is offering a change in the rational, accessible and clean energy; because it is one of the major concerns of the countries that signed the Kyoto treaty. That is why Colombia has a high potential in renewable resources, which should be aware of the proper and efficient use of these resources. The methodology is based on finding the most intense wind that occurs in the region of Huila department based on the research data it has collected on registration IDEAM for over 10 years.
\end{abstract}

Keywords: Meteorology; distribution; histogram; settings; wind rose; environmental impact

1 Msc. Ingeniero Electrónico. Universidad Surcolombiana. Av. Pastrana Carrera 1a. Neiva, Huila, Colombia. E-mail: jdavid@usco.edu.co

2 Msc. Ingeniero Electrónico. Universidad Surcolombiana. Av. Pastrana Carrera 1a. Neiva, Huila, Colombia. E-mail: julian.molina@usco.edu.co

3 Ingeniero Electrónico Universidad Surcolombiana. Av. Pastrana Carrera la. Neiva, Huila, Colombia. E-mail: jhonserra@usco.edu.co

Recibido: 1 agosto 2015. Aceptado: 30 mayo 2016. 


\section{Introducción}

La energía Eólica, es un recurso natural renovable poco explotado en nuestro país, pero ¿qué es la energía eólica?, como su nombre lo indica (El término eólico viene del latín Aeolicus, perteneciente o relativo a Eolo, dios de los vientos en la mitología griega) es la energía generada por efecto del viento a través de elementos conocidos como turbinas o aerogeneradores los cuales transforman la energía cinética del viento en energía eléctrica, este proceso se suele llevar a cabo en los parques eólicos (Molina \& Villarrubia, 2012).

Cuando se habla de parques eólicos, se hace mención a lugares donde se transforma y recolecta la energía generada por medio de las turbinas o aerogeneradores (EPM, 2010); estos elementos van montados en torres que miden entre 40 o 60 metros de altura y están en grupos que pueden variar desde 3 y hasta una decena de ellos. Pero para lograr crear un parque eólico en un sitio determinado se debe cumplir con unos requerimientos ambientales especiales para que su funcionamiento sea viable y la inversión aplicada sea recuperada de forma segura (Parra, 2012); (Gipe, 2012).

Según estudios que ha hecho el estado (Unidad de planeación Minero Energética, 2015), dentro del país hay diferentes lugares que cumplen con las características mínimas para la creación de uno de estos parques, en este caso se toma el punto de la estación "la Legiosa" ubicado en el departamento del Huila, municipio de Colombia, donde se hará el estudio de viabilidad técnicay económica para la creación de un parque eólico (Guzmán, 2013). El cual es importante destacar que la estación de la Legiosa es completamente diferente a las otras estaciones del departamento del Huila, ya que dentro de su territorio posee una velocidad promedio superior; este potencial eólico de la zona da cabida para presentar el estudio de viabilidad justificados en el proyecto, siendo así una buena oportunidad para el avance energético del departamento y un buen negocio para los inversores ya que gracias a la venta de esta energía como los incentivos que se recibirán del estado, se consigue recuperar la inversión en pocos años.

\section{Metodología}

En el desarrollo del estudio de viabilidad técnica se tomaron en cuenta los factores que influyen para la generación de energía eólica, siendo los más determinantes los datos estadísticos entregados por el IDEAM para el sitio conocido como la Legiosa en el departamento del Huila (municipio de Colombia). Se decidió el lugar de la Legiosa porque es el único punto en el interior de Colombia que brinda un potencial eólico relativamente elevado.

Para la recolección de los datos necesarios se hicieron los correspondientes trámites frente al Instituto de Hidrología, Meteorología y Estudios Ambientales de Colombia "IDEAM" los cuales tardaron alrededor de mes y medio para lograr obtener la información que fuera a utilizar este estudio (Atlas de los vientos, 2016); (Ingeominas, 2015).

La localización geográfica exacta la proporciono el instituto geográfico Agustín Codazzi "IGAC" por medio de la carta topográfica correspondiente a la región de Colombia Huila con la nomenclatura 303-IV-B por medio de la cual se descubre el relieve topográfico que presenta la región, en él se puede ver que la región que envía los vientos hacia este lugar son los llanos orientales y estos se concentran en el paso de la Legiosa hacia el interior de Colombia.

Según los datos entregados por el IDEAMlos vientos en el puno de la Legiosa se presentan de ESTE a OESTE con pequeñas variaciones hacia el NOR-ESTE en los meses de febrero y marzo y hacia SUR-ESTE en septiembre y octubre (Atlas de los vientos, 2016).

\section{Modelado matemático}

En este apartado se hará una descripción de los conceptos a tener en cuenta para los cálculos de la velocidad y potencia que son de relevancia para el diseño del parque eólico.

Perfil de viento: velocidad de viento como una función de la altura sobre el terreno. La forma de este perfil depende principalmente de la rugosidad del terreno.

Velocidad de viento potencial: se define como la velocidad del viento que se observaría en un terreno completamente planoy abierto (Busby, 2013); (Spilsbury, 2008), típicamente especificado para $10 \mathrm{~m}$, para este caso los datos que nos suministró el IDEAM, la altura es de $0 \mathrm{~m}$.

Para hallar la velocidad de viento actual (no potencial), se deben aplicar correlaciones a la velocidad de viento potencial, como la rugosidad del terreno (Pasqualetti, et al., 2009). Para hallar le referencia existente entre el incremento de velocidad y altura se utiliza la siguiente expresión (Moraleda, 2013). 


$$
\frac{V 1}{V 2}=\left[\frac{h 1}{h 2}\right]^{\propto}
$$

En esta ecuación 1, se define:

V1: la velocidad del viento a la altura $h 1$. $\mathrm{V}$ : la velocidad del viento a la altura $\mathrm{h} 2$. $\alpha$ : coeficiente de rugosidad.

Dado que la potencia eólica cambia con el cubo de la velocidad del viento, es posible estimar la potencia eólica aplicando la siguiente expresión: (Ecuación 2).

$$
\frac{P 1}{P 2}=\left[\frac{h 1}{h 2}\right]^{2 \propto}
$$

En la ecuación 2, se hace referencia a:

P1: potencia eólica específica a la altura h1.

P2: potencia eólica específica a la altura h2.

Posteriormente se analizó la turbulencia del viento y obstáculos. El viento al incidir alrededor de superficies muy rugosas presenta grandes cambios en velocidad y dirección. Esta turbulencia disminuye la entrega de potencia a un equipo eólico y puede conducir vibración indeseable.

La evaluación del recurso eólico se hace a partir de la recolección de fichas en una base de datos diurna, mensual y anual, de la estación La Legiosa para así realizar su respectiva evaluación y análisis.

Se inició con los datos suministrados por el IDEAM sobre las variaciones diurna - mensual (meses de junio y julio de 2014) y el informe de la variación del viento anual (base de datos de 10 años). Con la información de la variación diurna -mensual se realizó la construcción del histograma de velocidades (también llamado distribución de frecuencias de velocidad), el cual permite observar el número de horas que la velocidad del viento varia dentro de un intervalo. Luego se puede estimar la velocidad promedio mediante la siguiente formula:

$$
\bar{V}=\sum_{n=1}^{N} \frac{V i}{N}
$$

En esta ecuación 3, se define:

$\bar{V}$ : La velocidad promedio para un periodo $\mathrm{T}$ de estimación.

Vi: La velocidad promedio horaria.

$N$ : El número total de horas del periodo de estimación.
El número total de horas del periodo de estimación.

Con la información promedio horaria se realiza una estimación de la potencia eólica específica $\left(\mathrm{W} / \mathrm{m}^{2}\right)$ en el lugar a través de la siguiente expresión:

$$
\frac{\bar{P}}{A}=\frac{1}{2} \rho \frac{1}{N}\left[\sum_{1}^{N} V i^{3}\right]
$$

En esta ecuación 4, se hace referencia a:

\section{Potencia promedio}

$\bar{P}$ : Potencia promedio

A: Área perpendicular al viento medida en metros cuadrados.

$\rho$ : Densidad del aire $(1.2 \mathrm{Kg} / \mathrm{m} 2$ a nivel del mar).

Luego se obtiene la constante Ke que es la relación entre la potencia eólica total promedio durante un periodo estimado y la potencia eólica instantánea calculada con la velocidad promedio aplicando la siguiente expresión.

$$
K e=\frac{V^{3}}{\bar{V}}(5)
$$

Esta ecuación 5 es adicional a la distribución de frecuencia de velocidad del viento, también se suele representar en la distribución de duración de velocidad de viento (curva de frecuencia acumulada de viento). Esta nos permite determinar el número de horas que un valor de velocidad es excedido en la escala de tiempo de estimación. Con las distribuciones de frecuencias del viento y su correspondiente frecuencia acumulada, se hace una aproximación por una función de densidad de probabilidad para el histograma de velocidades. La función de densidad de probabilidad (fdp) que se utiliza es la distribución de Weibull cuya ecuación 6 está dada por la siguiente expresión.

$$
f(v)=\frac{\alpha}{\beta}\left(\frac{v}{\beta}\right)^{\alpha-1} \exp \left[-\left(\frac{v}{\beta}\right)^{\alpha}\right](6)
$$

Esta distribución es de dos parámetros « $\alpha$ « que es el parámetro de forma de la distribución y « $\beta »$ que es el parámetro de escala de la distribución de Weibull.

Para lograr modelar el comportamiento del viento con la distribución de Weibull se debe determinar sus parámetros con base en la información de vientos dada por el IDEAM, de esta última se busca encontrar la velocidad promedio y la desviación estándar. Conocidos estos valores se ajustan con las ecuación anteriormente planteada y se logra conocer los parámetros $\alpha$ y $\beta$ de la distribución que mejor se ajustan la información de viento. 
Otro parámetro a determinar con la distribución de Weibull es la distribución de frecuencia acumulada (Ecuación 7).

$$
f(v)=1-\exp \left\{-\left(\frac{v}{\beta}\right)^{\alpha}\right\}
$$

Su uso permite realizar de una manera más sencilla la conversión de energía eólica efectiva del sistema de aerogeneradores.

\section{Análisis de resultados}

\section{Evaluación del potencial eólico}

El principal objetivo del proyecto es evaluar la viabilidad técnica para la generación de potencial eólico en el departamento del Huila Municipio Colombia, la estimación de la potencia instalable y la producción energética del emplazamiento, suponiendo la instalación de aerogeneradores que se indica en el documento, la zona se encuentra a 1476 m.s.n.m. Las coordenadas UTM donde se encuentra el parque eólico son los que se muestran en la tabla 1.

Tabla 1. Coordenadas UTM parque eólico

\begin{tabular}{lc}
\hline UTM E $(\mathbf{m})$ & UTM N(m) \\
\hline $532705.54 \mathrm{~m} \mathrm{E}$ & $365295.21 \mathrm{~m} \mathrm{~N}$ \\
$532945.91 \mathrm{~m} \mathrm{E}$ & $365263.96 \mathrm{~m} \mathrm{~N}$ \\
$533006.07 \mathrm{~m} \mathrm{E}$ & $366195.85 \mathrm{~m} \mathrm{~N}$ \\
$533263.45 \mathrm{~m} \mathrm{E}$ & $366095.43 \mathrm{~m} \mathrm{~N}$ \\
\hline
\end{tabular}

\section{Análisis de datos meteorológicos}

Estación meteorológica: los datos fueron suministrados por el IDEAM, de la estación La Legiosa, ubicada en el Municipio Colombia, en las siguientes coordenadas UTM (Tabla 2).

Tabla 2. Coordenadas estación meteorológica

\begin{tabular}{lcc}
\hline & UTMX (m) & UTMY (m) \\
\hline Estación & $533002.46 \mathrm{~m} \mathrm{E}$ & $365961.23 \mathrm{~m} \mathrm{~N}$
\end{tabular}

Análisis del Periodo de Referencia: Se tomaron los datos entregados por el IDEAM y se extrajeron las gráficas siguientes para el análisis pertinente del potencial eólico, la figura 1 muestra los patrones de variación diurna (mes de junio) en la estación $\mathrm{La}$ Legiosa. En esta se ilustra los días cuya velocidad pro-

\section{Variaciones Diurnas de Viento - La Legiosa - Mes de Junio}

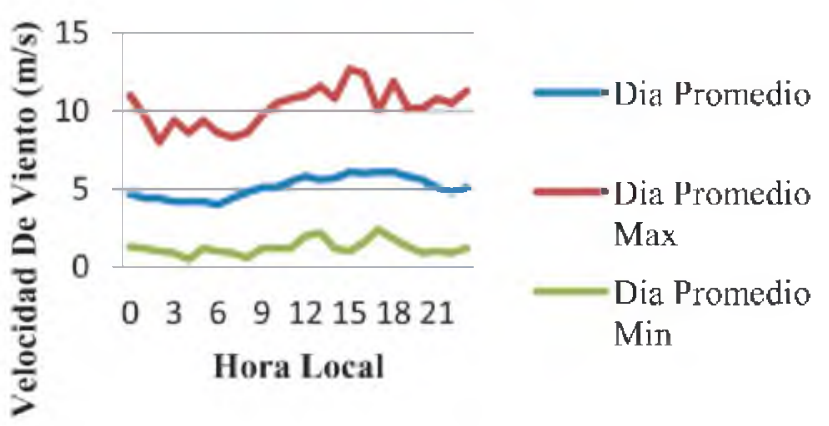

Figura 1. Análisis del periodo de referencia.

medio del viento diaria es máxima y mínima y el patrón de la hora-mes promedio (Buey, 2014).

\section{Velocidad de Viento Promedio}

En la figura 2 se presenta el patron de variacion promedio-dia a lo largo del mes de junio para la estacion La Legiosa.

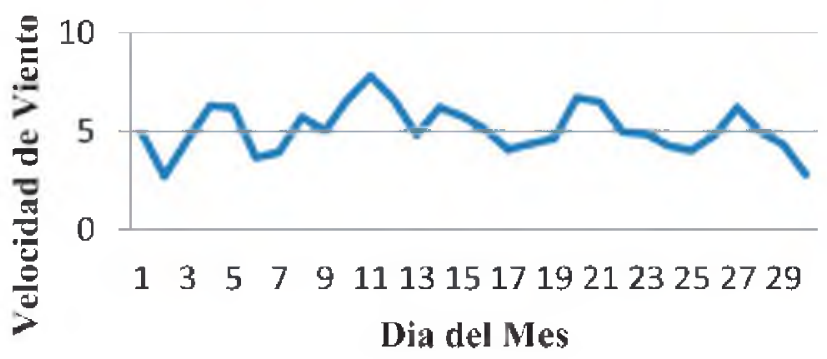

Figura 2. Velocidad del viento promedio diario a lo largo del mes de junio - La Legiosa.

En la figura 3 se muestra la variación de los vientos de 10 años entre los años 1985 y 1995.

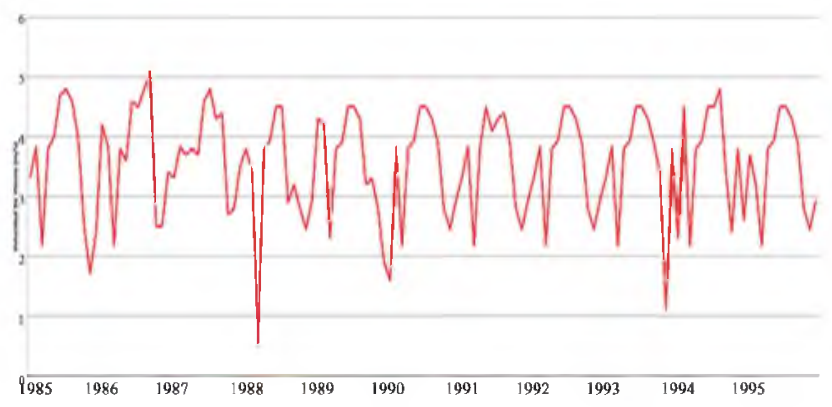

Figura 3. Variación de los vientos entre 1985 y 1995. 


\section{Histograma de Velocidades}

En la figura 4 se muestra el histograma de velocidades para la estación La Legiosa en el mes de junio (o también llamado distribución de frecuencias de velocidad).

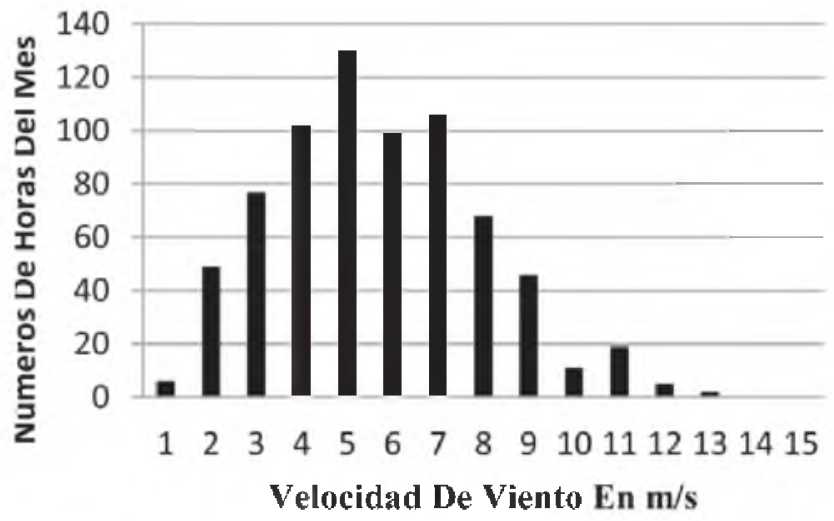

Figura 4. Histograma de velocidades - La Legiosa - Mes de junio.

\section{Frecuencia Acumulada de Velocidad de Viento}

En la figura 5 se representa la frecuencia acumulada de velocidad de viento, con la cual se logra determinar el numero de horas que un valor de velocidad es excedida en la escala de tiempo estimado.

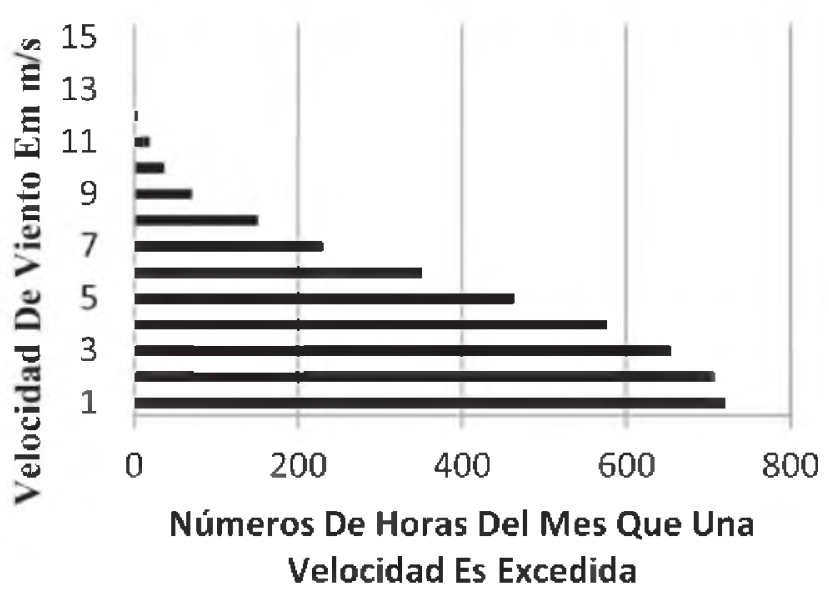

Figura 5. Frecuencia acumulada de velocidad del viento. La Legiosa - Junio.

\section{Rosa de las Magnitudes de Velocidades Medias}

En figura 6 esta rosa de magnitudes se puede observar la cantidad de veces que una velocidad se repite durante un mes, la velocidad media es $5.1 \mathrm{~m} / \mathrm{s}$.
ROSA DE VELOCIDADES MEDIAS DEL VIENTO

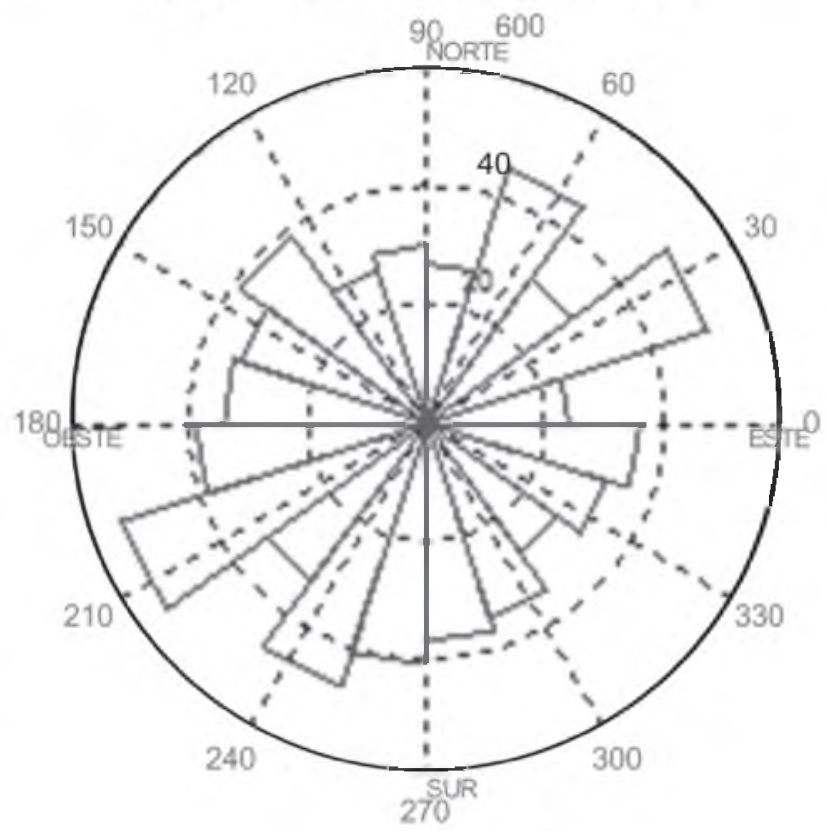

Figura 6. Rosa de las Magnitudes.

\section{Rosa De Direcciones Medias del Viento}

La figura 7 muestra la rosa de direcciones medias entrega la direccion predominante del viento durante un periodo de un mes o mas el viento predominante del este

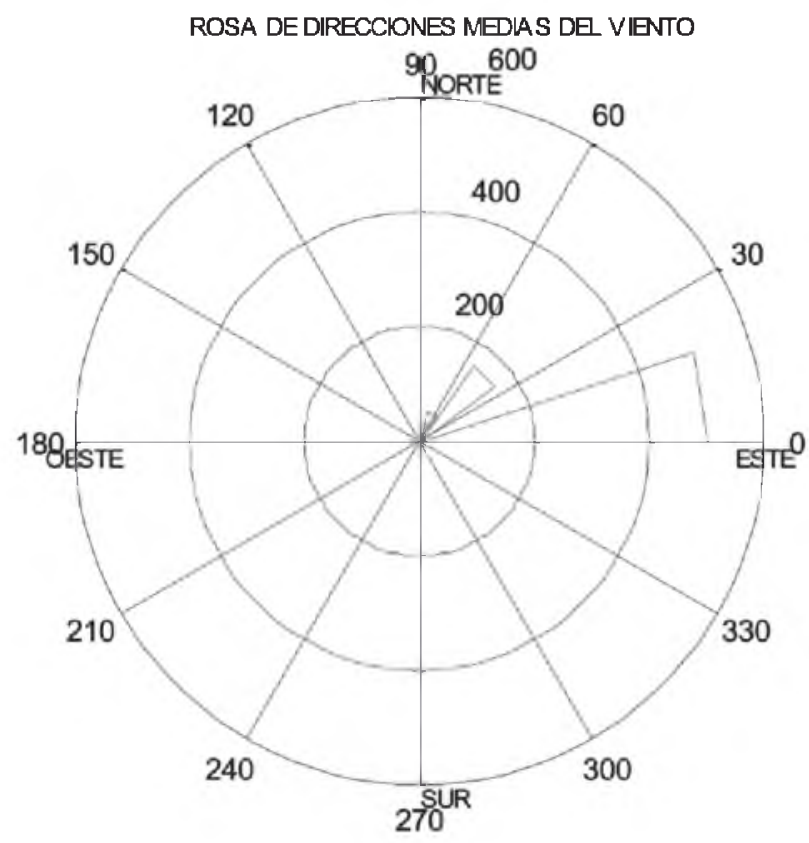

Figura 7. Rosa de direcciones medias de los vientos. 


\section{Rosa de Velocidady Dirección Media}

La figura 8 muestra la rosa de velocidades y direcciones media entrega durante un periodo de un mes, el viento predominante del este y la velocidad se ve representada por los colores.

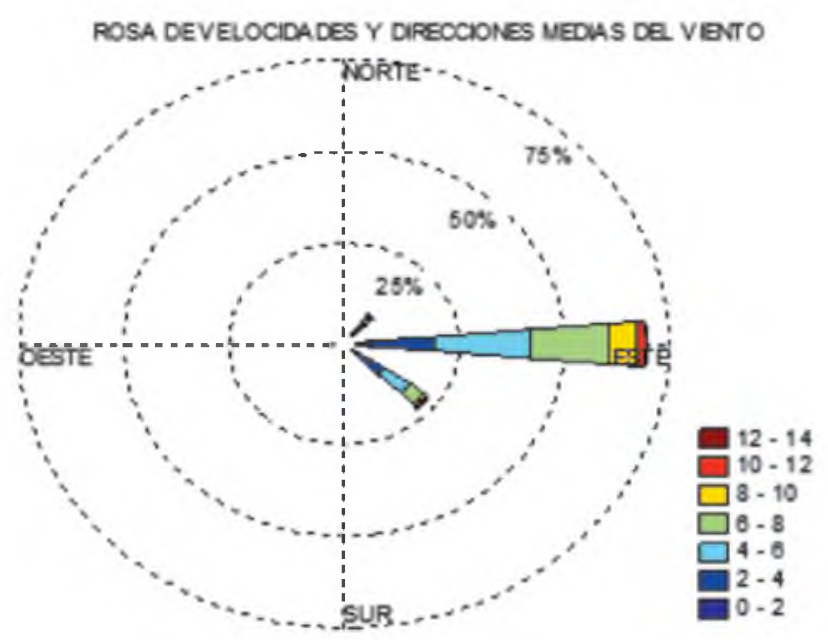

Figura 8. Rosa de velocidades medias y direcciones del Viento.

La figura 9 muestra la aplicación de la función de Densidad de probabilidad de la distribución de Weibull con los parámetros $\alpha=2.4 \mathrm{y} \mathrm{b}=6$.

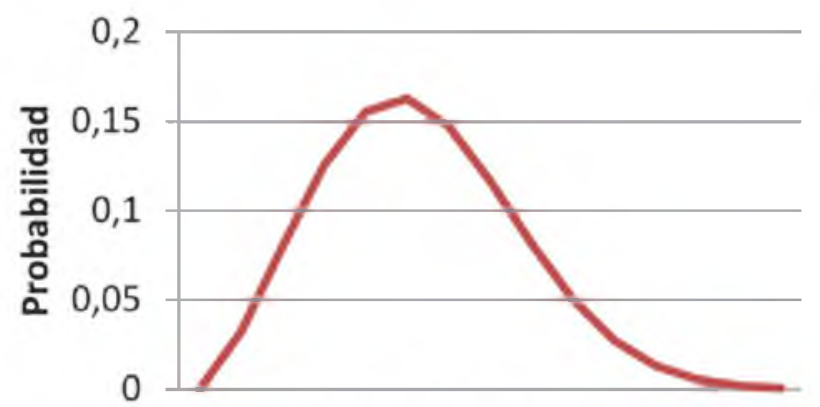

123456789101112131415

Velocidad De Vientos $(\mathrm{m} / \mathrm{s})$

Figura 9. Aplicación de la función de Densidad de probabilidad de la distribución de Weibull con los parámetros $\alpha=2.4 \mathrm{y} \mathrm{b}=6$.

En la figura 10 se aprecia la comparación entre el histograma de velocidades y el modelo matematico aproximado de la distribucion de weibull.

\section{Análisis del Periodo de Referencia}

A partir de las gráficas que nos proporcionan la velocidad media del viento durante el periodo de referencia

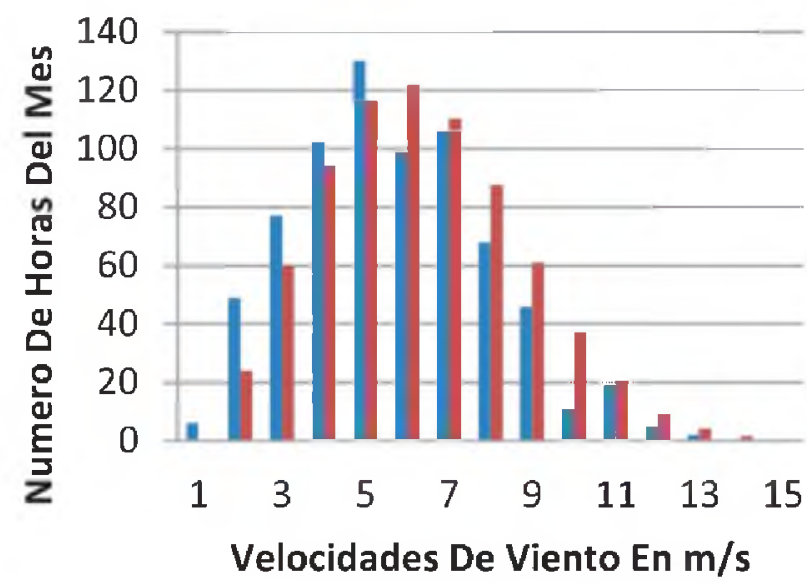

Figura 10. Histograma de velocidades y Distribución de Weibull - La Legiosa.

podemos deducir de la figura 11 que los aerogeneradores funcionan para una velocidad del viento superior a $3 \mathrm{~m} / \mathrm{s}$ e inferior a $21 \mathrm{~m} / \mathrm{s}$, de la figura anterior se deduce que los aerogeneradores estaran funcionando un $85.66 \%$ de las veces y una velocidad promedio de 5.1 para una altura de 10 metros; para un analisis de 80 metros la velocidad promedio es de $6.95 \mathrm{~m} / \mathrm{s}$ (Parra, 2012); (Saunder, 2008).

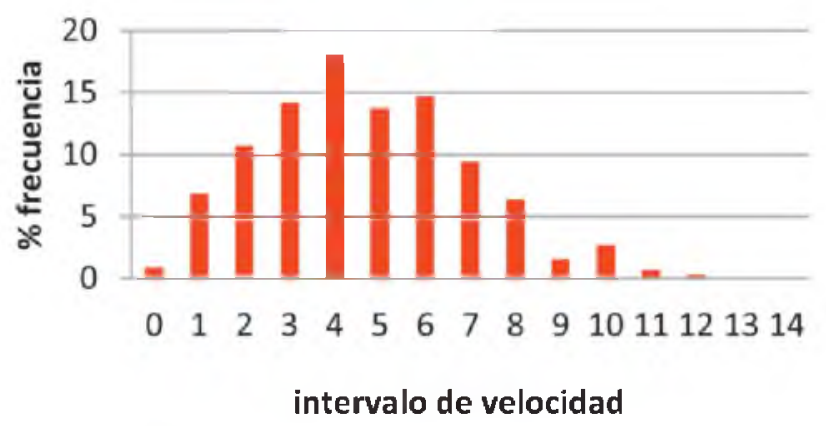

Figura 11. Frecuencias del Viento por intervalo de velocidad.

\section{Selección del aerogenerador}

Para la selección del aerogenerador se tienen en cuenta las condiciones climáticas de la región (Villarrubia, 2012); en donde se emplazará el parque eólico, la cual cuenta con una velocidad promedio de $6.95 \mathrm{~m} / \mathrm{s}$ y una temperatura ambiente que se encuentra en el rango de $5^{\circ} \mathrm{C}$ a $30^{\circ} \mathrm{C}$ y una densidad de aire de $1.12 \mathrm{~kg} / \mathrm{m}^{3}$

Con los datos anteriores podemos decir que los aerogeneradores que mejor se ajustan a la zona de trabajo son los aerogeneradores Gamesa g97 de 2 MW. Según datos del fabricante, en la siguiente figura 12 se ve la curva de potencia del aerogenerador G-97, lo se puede 


\section{Potencia}

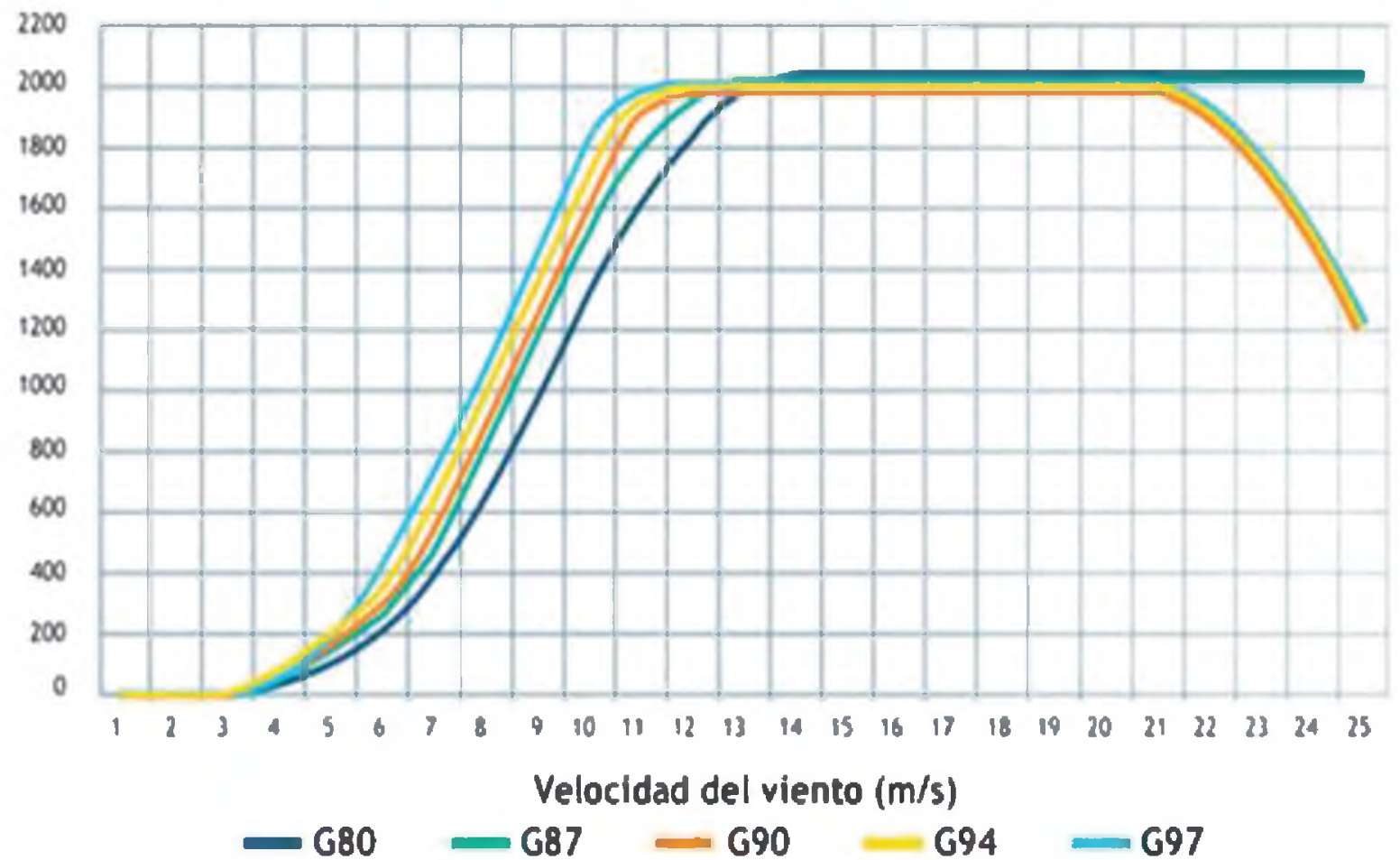

Figura 12. Curva de potencia del aerogenerador Gamesa G97.

deducir que para una velocidad promedio de $6.95 \mathrm{~m} / \mathrm{s}$, se obtendra una potencia de $900 \mathrm{KW} / \mathrm{h}$ (Gipe, 2012).

Con los resultados obtenidos, ya se puede calcular la producción neta del parque y las horas equivalentes de funcionamiento. Si un año hay 365 días, en un día 24 horas y los aerogeneradores estarán funcionando el $85.66 \%$ de las veces, un aerogenerador estará trabajando 7495,25 h/año. Para una velocidad media $6.95 \mathrm{~m} / \mathrm{s}$ la producción bruta será de: 6745,725 MWh/año. Teniendo en cuenta el rendimiento de $99 \%$ de los aerogeneradores y pérdidas del 6\% la producción neta de cada aerogenerador será de $6277,571685 \mathrm{MWh} /$ año. Por tanto, la producción neta del parque será de 25110,28674 MWh/ año que equivale a 3138,78 horas equivalentes como se observa en la tabla 3 .

\section{Conclusiones}

La iniciativa de realizar un estudio de viabilidad que demuestre la capacidad eólica que se encuentra sin explotar en este país, ayuda a abrir los caminos hacia la utilización de recursos renovables y fuentes de energía limpias, ya es interés del estado y las empresas privadas la explotación de este.

Las estadísticas elaboradas por el IDEAM deben de servir de punto de partida para la masificación de proyectos eólicos en Colombia y con ello disipar el impacto generado por la construcción de hidroeléctricas en caso más relevante la del Quimbo en Garzón ocasionando una considerable disminución en el trabajador del sector agrícola y masivas manifestaciones de las comunidades aledañas por los daños ocasionados.

Tabla 3. Potencia generada por el parque eólico

\begin{tabular}{lccccccc}
\hline $\begin{array}{l}\text { Horas de } \\
\text { funcionamiento/ } \\
\text { año }\end{array}$ & $\begin{array}{c}\text { Velocidad } \\
\text { media }(\mathbf{m} / \mathbf{s})\end{array}$ & $\begin{array}{c}\text { Producción } \\
\text { bruta } \\
(\mathbf{M W h} / \mathbf{a n ̃})\end{array}$ & $\begin{array}{c}\text { Rendimiento } \\
(\%)\end{array}$ & $\begin{array}{c}\text { Pérdidas } \\
(\%)\end{array}$ & $\begin{array}{c}\text { Producción } \\
\text { Neta } \\
\text { (MWh/año) }\end{array}$ & $\begin{array}{c}\text { Producción } \\
\text { Neta Parque } \\
\text { (MWh/año) }\end{array}$ & $\begin{array}{c}\text { Horas } \\
\text { Equivalentes }\end{array}$ \\
\hline 7495,25 & 6.395 & 6745,725 & 99 & 6 & 6277,57168 & 25110,2867 & 3138,78 \\
\hline
\end{tabular}


La viabilidad técnica para la construcción de un generador eólico puede calcular la producción neta del parque y las horas equivalentes de funcionamiento a un año con los aerogeneradores trabajando $7495,25 \mathrm{~h} /$ año a una velocidad media $6.95 \mathrm{~m} / \mathrm{s}$ la producción bruta será de: $6745,725 \mathrm{MWh} /$ año.

Teniendo en cuenta el rendimiento de $99 \%$ de los aerogeneradores y pérdidas del $6 \%$ la producción neta de cada aerogenerador será de 6277,571685 MWh/ año. Por tanto, la producción neta del parque será de 25110,28674 MWh/año.

De los factores elegidos en el análisis de sensibilidad, se tiene que la velocidad del viento tiene una influencia muy grande en la rentabilidad del proyecto. Una variación del $5 \%$ en este valor, hace que la rentabilidad varíe en un $\pm 1,5 \%$

Por todo lo expuesto, se entiende que el proyecto es viabley sostenible ambientalmente.

\section{Referencias bibliográficas}

Molina, J., Villarrubia, M., 2012. Viabilidad técnica y económica de un parque ecológico de 4400MW de potencia, Máster Interuniversitario UB-UPC d'Enginyeria en Energía, tesis de maestría.

Guzmán, C., 2013. Estudio de factibilidad para la construcción de un parque eólico en Colombia, Consultado el 6 de mayo de 2016. http://intellectum. unisabana.edu.co/bitstream/handle/10818/5036/ 130063. $P D F$ ? sequence $=1$ \&isAllowed $=y$

Atlas de los vientos y energías eólicas en Colombia., 2016. Consultado el 06 de mayo de 2016. http:// documentacion.ideam.gov.co/openbiblio/bvirtual/ 019813/Generalidades.pdf

Moraleda, J., 2013. Medida de desviación del viento, Consultado el 4 de mayo de 2016. http://www.upme. gov.co/Atlas_Viento.htm

Buey, E., 2014. Mapa de vientos, Consultado el 4 de mayo de 2016. http://www.upme.gov.co/Docs/ MapaViento/
Parra, J., 2012. Estudio de factibilidad económica y técnica para la implementación de un parque eólico sustentable en Arauca departamento de Arauca, Consultado el 4 de mayo de 2016. https://www. google.com.co/ url? $\mathrm{sa}=\mathrm{t} \& \mathrm{rct}=\mathrm{j} \& \mathrm{q}=\&$ esrc $=\mathrm{s} \&$ source $=$ web $\& \mathrm{~cd}=$ $8 \& \mathrm{cad}=$ rja $\&$ uact $=8 \&$ ved $=0$ ahUKEwiD $8 \mathrm{e}-\mathrm{qg} b$ NAhWL1h4KHVrfB1QQ FghLMAc\&url=http $\% 3 \mathrm{~A} \%$ $2 \mathrm{~F} \% 2 \mathrm{~F}$ reportes un bosque.edu.co $\% 2 \mathrm{~F}$ content $\%$ $2 \mathrm{~F} 2252 \% 2 \mathrm{Fshared}$ file $\% 2 \mathrm{~F} 3408 \&$ usg $=\mathrm{AFQjCNF} 0$ D08sX54jAZ_oXDGF9XQ e59phrA

Gipe, P., 2012. Wind power, Renewable energy for home, farm and business. The completely revised and expanded edition, $\mathrm{p} 97$

Unidad de Planeación Minero Energética., 2015. Formulación de un programa básico de normalización para aplicaciones de energías alternativas y difusión. En guía para la utilización de la energía eólica para generación de energía eléctrica Bogotá D.C, Colombia, $\mathrm{p} \mathrm{5-7}$

EPM, 2010. Experiencia en el Desarrollo de Proyectos de Energía Eólica, Consultado el 6 de mayo de 2016 .http://www.upme.gov.co/Eventos/ Foro_Normalizacion/Aplicacion $\% 20$ Eolica $\% 20$ EPM\%202008.pdf

Busby R, 2013, Wind Power, the industry grows up. Pennwell, p. 93

Spilsbury, R. L., 2008. The pros and cons of wind power. Susie Brooks, p. 42 .

Pasqualetti, M., Gipe, P., Righte, R., 2009. Wind Power in View: Energy Landscapes in a Crowded World. Academic press, p. 83.

Saunder, N., 2008. Energy for the Future and Global Warming: Wind Power: Easyread Large Edition, p. 44.

Villarrubia, M., 2012. Ingeniería de la Energía eólica. Marcombo, p. 283.

Ingeominas., 2015. Geología de Colombia, departamentos de Huila, Tolima y Meta. 